\title{
Lipid Compositions of Photomixotrophic Green Calluses and Chlorophyll Deficient Leaves of Tobacco
}

\author{
Toshiake Matsuzaki, Akira KoIwaI, Teruyoshi NaGaO, \\ Fumihiko SATO* and Yasuyuki YamadA* \\ Central Research Institute, \\ The Japan Tobacco \& Salt Public Corporation, \\ 6-2, Umegaoka, Midori-ku, Yokohama 227, Japan \\ *Research Center for Cell and Tissue Culture, Faculty of Agriculture, \\ Kyoto University, Sakyo-ku, Kyoto 606, Japan
}

Received August 8, 1983

\begin{abstract}
Among photomixotrophic green calluses tested (N. rustica. N. tobacum L. cv. BY-4 and Samsun), the callus of Samsun had the highest contents of chlorophyll and chloroplast lipids, such as monogalactosyldiglyceride (MGDG), digalactosyldiglyceride (DGDG), sulfoquinovosyldiglyceride (SQDG) and phosphatidylglycerol (PG). However, the chlorophyll and chloroplast lipids in the green callus of Samsun were still $1 / 6$ and $1 / 3$ of that in the parent leaves, respectively. The relative content of $\alpha$-linolenate in MGDG, DGDG and SQDG of the green calluses were higher than that of the white calluses. The ratios of hexadecatrienoate in MGDG and hexadecenoate $\left(\Delta^{3}\right.$-trans $)$ in PG in the green calluses were trace or less compared with that of the parent leaves. The crude lipids and total fatty acid contents of the chlorophyll deficient leaves $(N$. tabacum L. cv. Consolation 402 and Dominant Aurea Su/su) were almost the same as those of the normal leaves (cv. BY-4 and Samsun), although the chlorophyll contents of the chlorophyll deficient leaves were $1 / 3 \sim 1 / 4$ of that of the normal leaves. The ratios of chloroplast lipids in the total polar lipids in the chlorophyll deficient leaves were a little lower than that in the normal green leaves, but the former had a slightly higher ratio of phospholipids such as phosphatidylcholine and phosphatidylethanolamine than the latter. There were few differences in the fatty acid compositions of each individual lipid betweeen both types of leaves.
\end{abstract}

In recent years attempts to get highly chlorophyllous tissues of green calluses have been made by selecting strains with more chlorophyll tissues ${ }^{1)}$ and by growing green calluses photoautotrophically. ${ }^{1,2)}$ However, the chlorophyll content of green calluses was still low compared to their parent leaves. ${ }^{3)}$ This low chlorophyll content in the tissues generally is thought to reflect the low content of chloroplast lipids. ${ }^{4}$ One purpose of this report is to ascertain this relationship in green calluses of tobacco plants having different chlorophyll contents. On the other hand, among tobacco plants several chlorophyll deficient varieties, such as Nicotiana tabacum L. cv. Consolation 4025) and Dominant Aurea $\mathrm{Su} / \mathrm{su}^{6}{ }^{6)}$ are known. Although their chlorophyll contents are low, they grow normally and have higher photosynthetic activities per chlorophyll compared to the normal varieties. ${ }^{7)}$ Another purpose of this report is to compare the lipid profiles between green calluses and these chlorophyll deficient leaves. These studies should help in understanding the developmental features of chloroplasts in the green calluses in more

Abbreviations: NAA, $\beta$-Naphthaleneacetic acid; MGDG, monogalactosyldiglyceride; DGDG, digalactosyldiglyceride; SQDG, sulfoquinovosyldiglyceride; PG, phosphatidylglycerol; PC, phosphatidylcholine; PE, phosphatidylethanolamine; PI, phosphatidylinosytol; $16: 0$, palmitic acid; $16: 1, t, \Delta^{3}$-trans hexadecenoic acid; $16: 3$, hexadecatrienoic acid; $18: 0$, stearic acid; $18: 1$, oleic acid; $18: 2$, linoleic acid; $18: 3, \alpha$-linolenic acid; TLC, thin layer chromatography; GLC, gas liquid chromatography. 
detail. This paper deals with the comparison of the lipid contents and their fatty acid composition in leaves and calluses having different chlorophyll contents.

\section{MATERIALS AND METHODS}

Materials. Tobacco plants (Nicotiana rustica and Nicotiana tabacum L. cv. BY-4, Samsun, Consolation 402, and Dominant Aurea Su/su) were grown in soil in $0.02 \mathrm{~m}^{2}$ pots in a greenhouse. The plants were decapitated at the 14th week after sowing when they were at the onset of flowering, except for Dominant Aurea $\mathrm{Su} / \mathrm{su}$. The middle (18 to 19th leaves on the stalk from the cotyledons) leaves of four species and the upper (18 to 19th) leaves of Dominant Aurea Su/su were chosen for samples of the green leaves. Tobacco calluses (Nicotiana rustica and Nicotiana tabacum L. cv. BY-4), originally derived from the leaf in 1982 on the agar medium of Linsmaier-Skoog basal medium ${ }^{8)}$ with $10 \mu \mathrm{M}$ NAA, $1 \mu \mathrm{M}$ kinetin and doubled concentration of vitamins in the original solution. Calluses were subcultured 3 times at 14 day intervals on the above agar medium under dark or under $12 \mathrm{hr}$ photoperiod at a light intensity of 3000 lux. Other strains of tobacco calluses (highly chlorophyllous tobacco cells and white cells of Nicotiana tabacum L. cv. Samsun) subcultured at 14 day intervals for over 2 years $^{2)}$ on the liquid Linsmaier-Skoog basal medium with the same hormone and vitamin concentrations as mentioned above were analyzed. All tissues, including leaves with midribs removed, were freezedried, powdered and stored at $-20^{\circ} \mathrm{C}$ until extraction for total lipids.
Lipid extraction and polar lipid separation. Total lipids were extracted from powdered samples ( $1 \mathrm{~g}$ dry wt) with chloroform-methanol $(1: 2, \mathrm{v} / \mathrm{v})$ according to the method of Allen and Good, ${ }^{9)}$ and the lipid content was determined as described previously. ${ }^{10)}$ The total lipid solution was applied to silica gel TLC. Polar lipids were separated by developing with chloroform-methanol-water $(65: 25: 4$, $\mathrm{v} / \mathrm{v}$ ) in the first direction and with chloroform-methanolisopropylamine-ammonia $(65: 35: 0.5: 5, \mathrm{v} / \mathrm{v})$ in the second direction. Spots of lipids were located under UV after spraying with Rhodamine $6 \mathrm{G}$ solution. Each lipid was identified by cochromatography with standards and by spraying with specific reagents for the lipid class. ${ }^{11,12)}$

Lipid analyses. Individual separated lipids were methylated with $5 \% \mathrm{H}_{2} \mathrm{SO}_{4}$ in methanol, and analyzed by FIDGLC using glass columns packed with 5\% 1,4-butanediol glycol succinate polyester on Chromosorb W (DMCS). The column temperature was maintained at $205^{\circ} \mathrm{C}$ with a helium flow rate of $50 \mathrm{ml} / \mathrm{min}$. Methyl esters were identified by comparing the retention times with those reference methyl esters and by GC-MS (Hitachi M-80). The content of each polar lipid was calculated as its constituent fatty acids, and the value was converted to a molar basis. ${ }^{13)}$ The distribution of fatty acids in the $\mathrm{C}-1$ and $\mathrm{C}-2$ positions of the galactolipids and phospholipids were investigated by means of enzymic hydrolysis with the lipase from Rhizopus delemar. ${ }^{14)}$ The chlorophyll content was determined according to Arnon. ${ }^{15)}$

\section{RESULTS}

Table I shows the contents of crude lipids, total fatty acids, and their fatty acid com-

\section{Table I. Crude Lipids, Total Fatty Acids, and Fatty Acid Compositions in Leaves and Calluses of $N$. rustica AND SeVeral VARIETIES of $N$. tabacum}

\begin{tabular}{|c|c|c|c|c|c|c|c|c|c|c|c|}
\hline \multirow{2}{*}{ Tissue } & \multirow{2}{*}{ Species } & \multirow{2}{*}{$\begin{array}{l}\text { Crude lipids } \\
\qquad \begin{array}{l}\text { (mg/g } \\
\text { dry wt) }\end{array}\end{array}$} & \multirow{2}{*}{$\begin{array}{r}\text { Chlo } \\
\text { (mg/g } \\
\text { dry wt) }\end{array}$} & \multirow{2}{*}{$\begin{array}{l}\text { rophyll } \\
\left(\mathrm{mg} / 100 \mathrm{~cm}^{2}\right. \\
\text { leaf area) }\end{array}$} & \multirow{2}{*}{$\begin{array}{c}\text { Total fatty } \\
\text { acids } \\
\text { (mg/g dry wt) }\end{array}$} & \multicolumn{6}{|c|}{ Fatty acid composition $(\mathrm{mol} \%)$} \\
\hline & & & & & & $16: 0$ & $6: 1$, & $16: 3$ & $18: 0$ & $18: 1$ & $18: 218: 3$ \\
\hline \multirow[t]{6}{*}{ Leaf } & N. rustica & 100 & 12.0 & & 30.3 & 23.6 & 2.6 & 7.0 & 1.8 & 1.4 & 8.155 .5 \\
\hline & N. tabacum & & & & & & & & & & \\
\hline & BY -4 & 108 & 15.06 & 4.25 & 36.2 & 19.4 & 2.6 & 9.0 & 2.2 & 1.0 & 9.955 .9 \\
\hline & Samsun & 132 & 18.49 & 4.25 & 35.3 & 17.7 & 2.1 & 6.2 & 2.1 & 1.8 & 9.360 .8 \\
\hline & Consolation 402 & 100 & 5.18 & 1.23 & 34.1 & 14.5 & 2.9 & 8.6 & 1.4 & 1.7 & 11.759 .2 \\
\hline & Dominant Aurea $\mathrm{Su} / \mathrm{su}$ & 109 & 3.91 & 0.78 & 38.9 & 15.3 & 3.3 & 8.3 & 2.5 & 1.3 & 13.256 .1 \\
\hline \multirow[t]{7}{*}{ Callus } & N. rustica (green) & 31 & 0.10 & & 15.0 & 25.6 & 0.3 & $\operatorname{tr}$ & 4.0 & 3.9 & 48.617 .6 \\
\hline & (white) & 31 & - & & 13.9 & 25.6 & & & 4.1 & 4.6 & 51.114 .6 \\
\hline & N. tabacum & & & & & & & & & & \\
\hline & BY $-4 \quad$ (green) & 30 & 0.49 & & 15.7 & 22.9 & $\operatorname{tr}$ & $\operatorname{tr}$ & 5.1 & 1.4 & 34.236 .4 \\
\hline & (white) & 30 & - & & 13.5 & 25.7 & & & 5.8 & 2.2 & 47.518 .8 \\
\hline & (green) & 50 & 2.95 & & 17.6 & 25.7 & $\operatorname{tr}$ & $\operatorname{tr}$ & 2.6 & 0.5 & 31.639 .6 \\
\hline & (white) & 36 & - & & 14.6 & 23.8 & & & 2.1 & 1.8 & 51.021 .3 \\
\hline
\end{tabular}


Table II. Polar Lipid Distribution in Leaves and Calluses of $N$. rustica AND SeVeral VARIETIES OF $N$. tabacum

Polar lipid composition $(\mathrm{mol} \%)$

\begin{tabular}{|c|c|c|c|c|c|c|c|c|c|c|c|}
\hline \multirow{3}{*}{ Polar lipid } & \multicolumn{5}{|c|}{ Leaf } & \multicolumn{6}{|c|}{ Callus } \\
\hline & \multirow[t]{2}{*}{ N. rustica } & \multicolumn{4}{|c|}{ N. tabacum } & \multicolumn{2}{|c|}{ N. rustica } & \multicolumn{4}{|c|}{ N. tabacum } \\
\hline & & BY -4 & Samsun & $\begin{array}{c}\text { Consolation } \\
402\end{array}$ & $\begin{array}{c}\text { Dominant } \\
\text { Aurea } \mathrm{Su} / \mathrm{su}\end{array}$ & Green & White & $\begin{array}{l}\text { BY-4 } \\
\text { Green }\end{array}$ & White & $\begin{array}{l}\text { Samsun } \\
\text { Green }\end{array}$ & White \\
\hline MGDG & 35.1 & 38.3 & 42.1 & 34.3 & 34.7 & 2.9 & 3.1 & 9.1 & 4.1 & 22.2 & 2.8 \\
\hline DGDG & 29.1 & 27.6 & 26.8 & 24.6 & 16.8 & 5.5 & 5.7 & 6.0 & 5.6 & 21.2 & 10.7 \\
\hline SQDG & 8.6 & 7.7 & 6.7 & 7.9 & 6.9 & 1.1 & 0.7 & 2.2 & 1.4 & 4.9 & 1.5 \\
\hline PG & 7.6 & 7.8 & 7.4 & 7.9 & 10.2 & 4.8 & 4.1 & 3.4 & 2.8 & 5.7 & 3.1 \\
\hline $\mathrm{PC}$ & 10.4 & 5.8 & 8.3 & 10.0 & 16.2 & 45.0 & 44.1 & 44.6 & 49.6 & 22.2 & 37.0 \\
\hline $\mathrm{PE}$ & 3.7 & 3.0 & 3.5 & 5.4 & 7.5 & 23.5 & 24.4 & 16.7 & 12.0 & 12.8 & 27.7 \\
\hline Others $^{a}$ & 5.5 & 9.8 & 5.2 & 9.9 & 7.7 & 17.2 & 17.9 & 18.0 & 24.5 & 11.0 & 17.2 \\
\hline \multicolumn{12}{|c|}{ Fatty acids of MGDG + DGDG + SQDG + PG (mg/g dry wt) } \\
\hline & 15.42 & 18.12 & 17.63 & 15.38 & 16.76 & 1.51 & 1.46 & 1.55 & 0.76 & 5.30 & 1.24 \\
\hline \multicolumn{12}{|c|}{ Fatty acids of $\mathrm{MGDG}+\mathrm{DGDG}+\mathrm{SQDG}+\mathrm{PG}\left(\mathrm{mg} / 100 \mathrm{~cm}^{2}\right.$ leaf area $)$} \\
\hline & & 5.10 & 4.05 & 3.66 & 3.37 & & & & & & \\
\hline \multicolumn{12}{|c|}{ Total polar lipid fatty acids (mg/g dry wt) } \\
\hline & 19.18 & 22.26 & 21.24 & 20.59 & 24.43 & 10.57 & 10.72 & 7.48 & 5.48 & 9.81 & 6.83 \\
\hline
\end{tabular}

${ }^{a}$ PI, phosphatidic acid and diphosphatidylglycerol. 
Table III. Fatty Acid Composition of Each Polar Lipid In Leaves and CAlluses of $N$. tabacum and $N$. rustica

\begin{tabular}{|c|c|c|c|c|c|c|c|c|}
\hline \multirow{2}{*}{$\begin{array}{l}\text { Polar } \\
\text { lipid }\end{array}$} & \multirow{2}{*}{ Tissue species } & \multicolumn{7}{|c|}{ Fatty acid composition $\left(\mathrm{mol}^{\%}\right)$} \\
\hline & & $16: 0$ & $16: 1, \mathrm{t}$ & $16: 3$ & $18: 0$ & $18: 1$ & $18: 2$ & $18: 3$ \\
\hline \multirow[t]{11}{*}{ MGDG } & Leaf $N$. tabacum & & & & & & & \\
\hline & BY-4 & 2.1 & & 18.6 & $\operatorname{tr}$ & $\operatorname{tr}$ & 3.6 & 75.7 \\
\hline & Consolation 402 & 1.9 & & 18.8 & $\operatorname{tr}$ & $\operatorname{tr}$ & 1.9 & 77.5 \\
\hline & Dominant Aurea Su/su & 1.3 & & 20.6 & $\operatorname{tr}$ & $\operatorname{tr}$ & 1.8 & 76.3 \\
\hline & Callus $N$. rustica (green) & 12.0 & & & 3.5 & 2.9 & 24.0 & 57.6 \\
\hline & (white) & 10.7 & & & 2.9 & 4.7 & 34.0 & 47.7 \\
\hline & N. tabacum & & & & & & & \\
\hline & BY-4 (green) & 7.8 & & $\operatorname{tr}$ & 1.8 & 0.9 & 15.5 & 74.0 \\
\hline & (white) & 18.8 & & & 5.2 & 4.0 & 29.4 & 42.6 \\
\hline & Samsun (green) & 5.1 & & $\operatorname{tr}$ & 0.9 & 0.8 & 16.5 & 76.7 \\
\hline & (white) & 16.7 & & & 2.0 & 1.6 & 37.1 & 42.6 \\
\hline \multirow[t]{11}{*}{ DGDG } & Leaf $N$. tabacum & & & & & & & \\
\hline & BY-4 & 15.7 & & 2.2 & 1.6 & 0.4 & 3.7 & 76.4 \\
\hline & Consolation 402 & 11.4 & & 2.7 & 0.9 & 1.3 & 2.5 & 81.2 \\
\hline & Dominant Aurea $\mathrm{Su} / \mathrm{su}$ & 17.2 & & 2.6 & 1.4 & 1.7 & 3.1 & 74.0 \\
\hline & Callus $N$. rustica (green) & 33.0 & & & 8.8 & 3.8 & 25.0 & 29.4 \\
\hline & (white) & 27.5 & & & 7.5 & 5.7 & 33.3 & 26.0 \\
\hline & N. tabacum & & & & & & & \\
\hline & BY-4 (green) & 50.5 & & & 14.6 & 2.8 & 8.8 & 23.3 \\
\hline & (white) & 51.9 & & & 20.3 & 4.4 & 14.7 & 8.7 \\
\hline & Samsun (green) & 27.1 & & & 4.1 & 1.0 & 16.6 & 51.2 \\
\hline & (white) & 38.6 & & & 4.7 & 3.2 & 32.5 & 21.0 \\
\hline \multirow[t]{11}{*}{ SQDG } & Leaf $N$. tabacum & & & & & & & \\
\hline & BY-4 & 49.9 & & & 2.8 & 0.6 & 5.7 & 41.0 \\
\hline & Consolation 402 & 45.3 & & & 1.9 & 1.2 & 4.1 & 47.5 \\
\hline & Dominant Aurea Su/su & 55.4 & & & 2.2 & 1.5 & 4.6 & 36.3 \\
\hline & Callus $N$. rustica (green) & 42.1 & & & 7.1 & 5.6 & 25.8 & 19.4 \\
\hline & (white) & 50.2 & & & 8.8 & 6.4 & 24.3 & 10.3 \\
\hline & N. tabacum & & & & & & & \\
\hline & BY-4 (green) & 55.4 & & & 10.4 & 2.9 & 10.6 & 20.7 \\
\hline & (white) & 75.7 & & & 14.5 & 1.2 & 3.7 & 4.9 \\
\hline & Samsun (green) & 49.1 & & & 4.9 & 2.0 & 15.8 & 28.2 \\
\hline & (white) & 45.0 & & & 4.8 & 4.6 & 26.6 & 19.0 \\
\hline \multirow[t]{11}{*}{ PG } & Leaf $N$. tabacum & & & & & & & \\
\hline & BY -4 & 22.2 & 34.8 & & 2.1 & 3.9 & 11.7 & 25.3 \\
\hline & Consolation 402 & 15.8 & 34.8 & & 1.5 & 4.9 & 10.7 & 32.3 \\
\hline & Dominant Aurea $\mathrm{Su} / \mathrm{su}$ & 17.6 & 32.6 & & 1.0 & 2.7 & 10.0 & 36.1 \\
\hline & Callus $N$. rustica (green) & 33.8 & & & 2.1 & 2.9 & 44.1 & 17.1 \\
\hline & (white) & 32.7 & & & 2.0 & 3.1 & 47.2 & 15.0 \\
\hline & N. tabacum & & & & & & & \\
\hline & BY-4 (green) & 76.7 & $\operatorname{tr}$ & & 8.5 & 2.3 & 8.2 & 4.3 \\
\hline & (white) & 87.8 & & & 8.5 & 0.6 & 1.9 & 1.2 \\
\hline & Samsun (green) & 55.8 & 6.5 & & 4.5 & 7.4 & 17.3 & 8.5 \\
\hline & (white) & 33.2 & & & 1.3 & 1.5 & 43.2 & 20.8 \\
\hline
\end{tabular}

positions in leaves and calluses of tobacco plants having different chlorophyll contents. The chlorophyll contents of green calluses of $N$. rustica and $N$. tabacum L. cv. BY-4 and Samsun were $0.8,3.2$, and $15.9 \%$, respectively, of the parent leaves. The contents of crude lipids and total fatty acids of both green and white calluses were much smaller than those of the parent leaves. The green calluses had slightly higher contents of fatty acids than the 
TABLE III. (continued).

\begin{tabular}{|c|c|c|c|c|c|c|c|c|}
\hline \multirow{2}{*}{$\begin{array}{l}\text { Polar } \\
\text { lipid }\end{array}$} & \multirow{2}{*}{ Tissue species } & \multicolumn{7}{|c|}{ Fatty acid composition $(\mathrm{mol} \%)$} \\
\hline & & $16: 0$ & $16: 1, t$ & $16: 3$ & $18: 0$ & $18: 1$ & $18: 2$ & $18: 3$ \\
\hline \multirow[t]{11}{*}{$\mathrm{PC}$} & Leaf $N$. tabacum & & & & & & & \\
\hline & BY -4 & 29.7 & & & 7.0 & 2.3 & 28.3 & 32.7 \\
\hline & Consolation 402 & 22.5 & & & 4.0 & 4.8 & 38.5 & 30.2 \\
\hline & Dominant Aurea $\mathrm{Su} / \mathrm{su}$ & 22.8 & & & 4.8 & 2.3 & 34.7 & 35.4 \\
\hline & Callus $N$. rustica (green) & 25.8 & & & 4.5 & 5.1 & 50.4 & 14.2 \\
\hline & (white) & 26.6 & & & 4.2 & 5.5 & 51.2 & 12.5 \\
\hline & N. tabacum & & & & & & & \\
\hline & BY-4 (green) & 30.6 & & & 8.8 & 1.3 & 35.3 & 24.0 \\
\hline & (white) & 30.3 & & & 9.4 & 2.9 & 44.3 & 13.1 \\
\hline & Samsun (green) & 28.1 & & & 4.7 & 0.9 & 42.5 & 23.8 \\
\hline & (white) & 21.7 & & & 2.4 & 2.4 & 56.3 & 17.2 \\
\hline \multirow[t]{11}{*}{$\mathrm{PE}$} & Leaf $N$. tabacum & & & & & & & \\
\hline & BY -4 & 34.4 & & & 6.5 & 1.6 & 33.0 & 24.5 \\
\hline & Consolation 402 & 28.0 & & & 4.6 & 3.8 & 42.2 & 21.4 \\
\hline & Dominant Aurea $\mathrm{Su} / \mathrm{su}$ & 30.2 & & & 4.7 & 1.5 & 35.6 & 28.0 \\
\hline & Callus $N$. rustica (green) & 26.4 & & & 3.5 & 2.7 & 55.4 & 12.0 \\
\hline & (white) & 27.0 & & & 3.5 & 3.3 & 55.6 & 10.6 \\
\hline & N. tabacum & & & & & & & \\
\hline & BY $-4 \quad$ (green) & 32.7 & & & 7.3 & 1.1 & 41.4 & 17.5 \\
\hline & (white) & 30.4 & & & 6.5 & 2.4 & 48.9 & 11.8 \\
\hline & Samsun (green) & 24.7 & & & 2.6 & 1.8 & 50.4 & 20.5 \\
\hline & (white) & 23.3 & & & 1.9 & 1.4 & 59.8 & 13.6 \\
\hline
\end{tabular}

white calluses. The ratios of $18: 3$ in the callus tissues $(15 \sim 40 \%$ of total fatty acid contents) were also smaller than the parent leaves $(56 \sim 61 \%)$. Comparing the fatty acid compositions between the green calluses and the white calluses, the former had a higher ratio of $18: 3$ and a lower ratio of $18: 2$. On the other hand, the crude lipid and total fatty acid contents of the chlorophyll deficient leaves $(N$. tabacum L. cv. Consolation 402 and Dominant Aurea $\mathrm{Su} / \mathrm{su}$ ) were almost the same as that of the normal leaves (cv. BY-4 and Samsun), although the chlorophyll contents per $\mathrm{g}$ dry wt of the chlorophyll deficient varieties were $1 / 3 \sim 1 / 4$ of that of the normal varieties. The fatty acid compositions of the chlorophyll deficient leaves almost resembled the normal green leaves. In both types of leaves $18: 3$ predominated $(56 \sim 59 \%)$, followed by $16: 0(15 \%)$ and $18: 2(12 \sim 13 \%)$.

Table II shows the contents of the total polar lipid fatty acids and the distributions of the individual polar lipids in leaves and cal- luses of tobacco plants. In spite of green or white culture, the calluses had fairly low contents of total polar lipids compared to those of the parent leaves. Nonchlorophyllous white calluses contained very small amounts of chloroplast lipids (i.e. MGDG, DGDG, SQDG and PG). Among green calluses, the callus of Samsun had the highest chloroplast lipids. However, the overall amount was about $1 / 3$ of that in the parent leaves. There was little difference in the total polar lipid fatty acid content between the chlorophyll deficient leaves and the normal green leaves $(21 \sim 24 \mathrm{mg} / \mathrm{g}$ dry $\mathrm{wt})$. The proportion of total chloroplast lipids in the total polar lipids in Consolation 402 and Dominant Aurea $\mathrm{Su} / \mathrm{su}$ was a little lower than that in normal leaves, but the proportion of phospholipids such as PC and PE in those varieties was a little higher. The ratio of chlorophyll to chloroplast lipids was below 0.5 in the chlorophyll deficient leaves and about 1.0 in the normal leaves.

The fatty acid compositions of the indi- 
vidual polar lipids in leaves and calluses of tobacco plants are shown in Table III. There were not any large differences found in the fatty acid composition of individual lipids among normal green leaves ( $N$. rustica, $N$. tabacum L. cv. Samsun and BY-4), so the data for BY-4 leaf only were shown. There were few differences between the chlorophyll deficient leaves and the normal leaves in the fatty acid composition of each polar lipid. MGDG and DGDG in the chlorophyll deficient leaves were also highly desaturated to $18: 3(74 \sim 82 \%)$. SQDG and PG in these leaves were also rich in $18: 3(32 \sim 48 \%)$. The $16: 3$ in MGDG and the $16: 1, t$ in PG were also detected in the chlorophyll deficient leaves in considerable amounts.

Generally, the ratio of $18: 3$ in MGDG, DGDG, and SQDG of the green calluses was higher than those of the white calluses. Especially, the ratios of 18:3 in MGDG of the green calluses of BY-4 and Samsun were almost equal to that in the parent leaves.
However, the ratios of 18:3 in DGDG, SQDG and PG in the green calluses were yet much lower than that in the parent leaves. The $16: 3$ and $16: 1, t$ being characteristic for MGDG and $\mathrm{PG}$, respectively, in the green leaves of tobacco were detected in a trace amount or a little in the green calluses. These specific fatty acids in MGDG and PG are known to be synthesized in the chloroplast, ${ }^{16)}$ and the amount of $16: 1, \mathrm{t}$ in PG especially reflects the developmental stages of thylakoids during greening. From this point of view, the chloroplast development in the green calluses seem still undifferentiated compared with that in the leaves. PC and PE, which are mainly included in other organelles than chloroplasts, were characterized by having relatively higher ratios of $18: 2,18: 3$, and $16: 0$ in both calluses and leaves. The ratio of $18: 3$ in PC and PE in the leaves was a little higher than that in the calluses.

Figure 1 shows the positional distributions of fatty acids between $\mathrm{C}-1$ and $\mathrm{C}-2$ at the
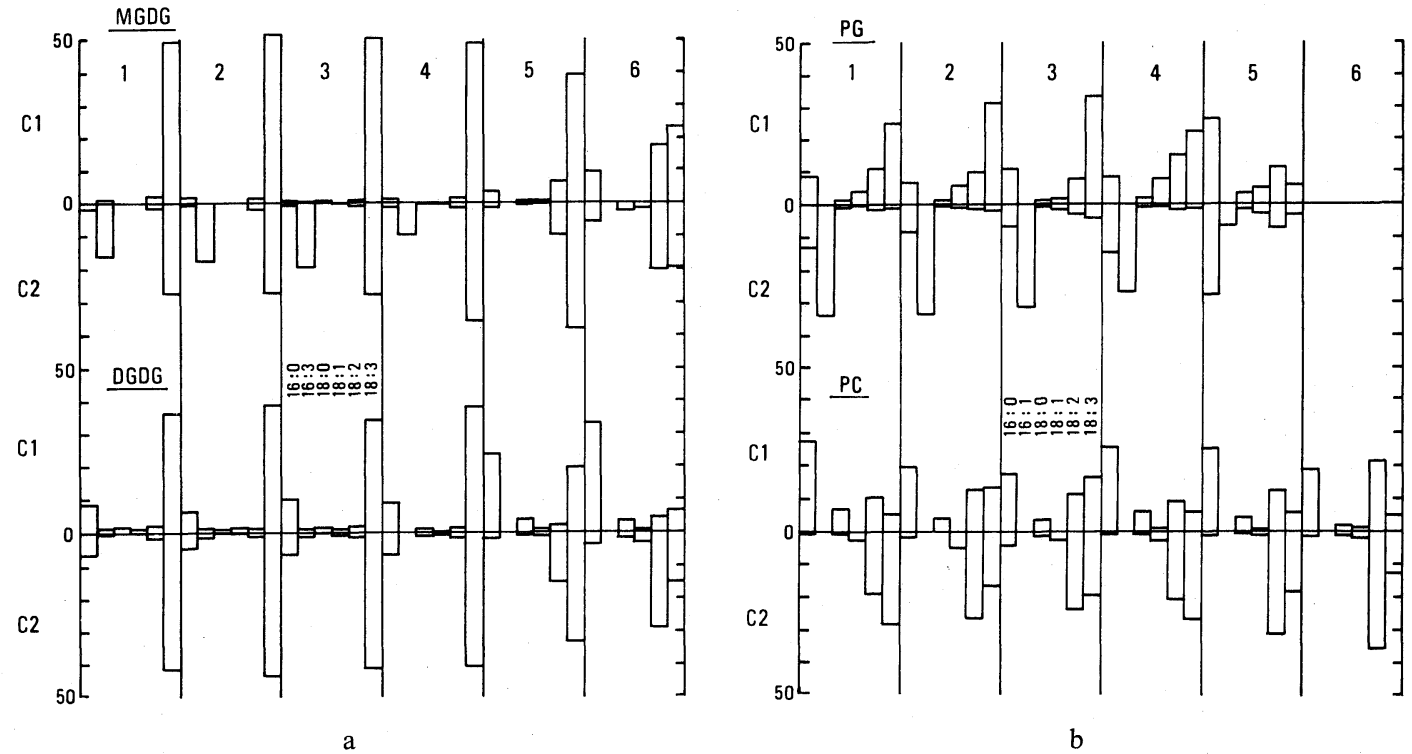

Figs. 1a and 1b. Positional Distributions ( $\mathrm{mol} \%$ ) of Fatty Acids between C-1 and C-2 at the Glycerol Moiety of MGDG and DGDG (Fig. 1a) and PG and PC (Fig. 1b) from Leaves (BY-4-1, Consolation 402-2, Dominant Aurea Su/su -3, Samsun -4), Green Callus of Samsun (5) and White Callus of Samsun (6).

Bars from left to right in MGDG and DGDG are 16:0,16:3,18:0,18:1,18:2 and 18:3, and bars from left to right in PG and PC are 16:0,16:1, 18:0,18:1, 18:2 and 18:3. PG in the white callus of Samsun (6) was not determined. 
glycerol moiety of MGDG, DGDG, PG and PC from leaves ( $N$. tabacum L. cv. BY-4, Samsun, Consolation 402 and Dominant Aurea Su/su) and calluses of Samsun. Little differences were found in the patterns of fatty acid distributions in the glycerol moiety in each lipid between the chlorophyll deficient leaves and the normal green leaves. Its patterns in the green callus of Samsun were a little different from that in the parent leaf. The former had less 16:3 in MGDG at the C-2 position, and also had a little $16: 1, \mathrm{t}$ in $\mathrm{PG}$ at the $\mathrm{C}-2$ position, although the latter had these fatty acids at the C-2 position with relatively high ratios. Comparing the green callus with the white callus of Samsun, the former had higher ratios of $18: 3$ in MGDG and DGDG at both the $\mathrm{C}-1$ and $\mathrm{C}-2$ position than the latter. The positional distribution patterns of fatty acids in PC were similar between the calluses and the leaves. The 16:0 in PC from each tissue was localized at $\mathrm{C}-1$, and the $18: 2$ and $18: 3$ were found more at C-2 than at C-1. These results are in agreement with those of Sieberz and Heinz. ${ }^{17)}$

\section{DISCUSSION}

Non-photosynthetic tissues such as roots, ${ }^{18)}$ etiolated leaves grown in the dark, ${ }^{19)}$ and heterotrophic white calluses contain smaller amounts of chloroplast lipids than photosynthetic green tissues. ${ }^{13,20)}$ On the other hand, during greening marked increases in chloroplast lipids, accompanied by the increases of chlorophyll and linoleate, have been observed in many plants. ${ }^{21}$ 23) With variegated tobacco leaves the white parts contained less chlorophyll and chloroplast lipids than the green parts. ${ }^{24)}$ Furthermore, various flower organs of tobacco such as petal, ovary, anther and pistil contains less chlorophyll and chloroplast lipids than leaf as well as less linolenate. ${ }^{25)}$ All the above results seem to show that the contents of chlorophyll are closely correlated with that of chloroplast lipids and linolenates. Our results obtained by analyzing the green calluses are in agreement with this.
However, the results obtained from the lipid analyses of chlorophyll deficient leaves seem to be inconsistent with the above hypothesis. Furthermore, electronmicroscopic observation showed that the chlorophyll deficient leaves had fewer grana stackings than the normal varieties. $^{7)}$

Then the comparison of the amounts of chloroplast lipids per g dry wt among leaves does not seem to reflect the electronmicroscopic observation. This discrepancy might be explained by comparing the amounts of chloroplast lipids per leaf area $\left(100 \mathrm{~cm}^{2}\right)$, that is, the chlorophyll deficient leaves had lower chloroplast lipids per leaf area than the normal varieties (Table II).

In spite of this, the amounts of chloroplast lipids in chlorophyll deficient leaves per unit of leaf area are much higher than that of chlorophyll. Concerning the chlorophyll pigments, we distinguish between light-harvesting pigments and light-converting pigments e.g., photosynthetic units and its reaction center. Schmid showed that Dominant Aurea $\mathrm{Su} / \mathrm{su}$ had approximately five times more photosynthetic activities per $\mathrm{mg}$ chlorophyll than normal varieties had. ${ }^{26}$ ) This led him to propose the idea that chlorophyll deficient varieties such as Dominant Aurea $\mathrm{Su} / \mathrm{su}$ had more reaction centers in the thylakoids than the normal varieties. If this hypothesis is true in the mutant varieties, the photosynthetic apparatus in the chlorophyll deficient leaves functions well to transfer light energy effectively to produce NADPH, ATP, or a high energy intermediate (state) assosiated with photophosphorylation. The latter intermediate is thought to have the main role with light in accelerating the fatty acid synthesizing system of chloroplasts. ${ }^{27)}$

On the other hand, the photomixotrophic cells (cv. Samsun) had lower photosynthetic activities $(94.5 \mu \mathrm{mol} \mathrm{CO} 2 / \mathrm{mg}$ chlorophyll $/ \mathrm{hr})$ than the photoautotrophic cells $(132.0 \mu \mathrm{mol}$ $\mathrm{CO}_{2} / \mathrm{mg}$ chlorophyll/hr) ${ }^{28)}$ or mesophyll cells from leaves of Papaver somniferum (110 $175 \mu \mathrm{mol} \quad \mathrm{CO}_{2} / \mathrm{mg}$ chlorophyll/hr). ${ }^{29)}$ These suggest that the photomixotrophic tobacco 
cells does not yet have well developed systems of photosynthesis and lipid biosynthesis compared with the chlorophyll deficient leaves or normal green leaves. In addition, the chloroplasts in the photomixotrophic cells of BY-4 had fewer grana stackings than that of Samsun or chloroplasts in the parent leaf (unpublished). These structural differences of thylakoids strongly reflect the chloroplast lipid content and composition of the cells.

In the green calluses of Chenopodium rubrum more chlorophyllous and more chloroplast rich tissues have been obtained by subculturing calluses from the photomixotrophic culture to the autotrophic culture. ${ }^{30)}$ Although photoautotrophic cultures of tobacco have succeeded, their chlorophyll content is still lower than that of the parent leaf. ${ }^{2)}$ The contents of chloroplast lipids, especially the contents of $16: 3$ in MGDG and 16:1, $t$ in PG in these tissues would give information on the developmental features of the chloroplast. And the $\mathrm{Su}$ gene of Dominant Aurea $\mathrm{Su} / \mathrm{su}$ might also give hints on how to select callus strains of tobacco having high photosynthetic activities.

\section{REFERENCES}

1) W. Hüsemann and W. Barz, Physiol. Plant., 40, 77 (1977).

2) Y. Yamada and F. Sato, Plant \& Cell Physiol., 19, 4 (1978).

3) D. R. Thomas and A. K. Stobart, J. Exp. Bot., 21, 274 (1970)

4) S. S. Radwan and H. K. Mangold, Adv. Lipid Res., 14, 171 (1976).

5) Y. Wada, Bull. Hatano Tob. Exp. Sta., 73, 1 (1973).

6) L. Burk and H. A. Menser, Tobacco Sci., 8, 101 (1964).

7) P. H. Homann and G. H. Schmid, Plant Physiol., 42, 1619 (1967).
8) F. Linsmaier and F. Skoog, Physiol. Plant., 18, 100 (1965).

9) C. F. Allen and P. Good, Methods in Enzymol., 23, 523 (1971).

10) A. Koiwai and T. Kisaki, Agric. Biol. Chem., 43, 597 (1979).

11) T. Yamakawa, R. Irie and M. Iwanaga, J. Biochem., 48, 490 (1960).

12) J. C. Dittmer and R. L. Lester, J. Lipid Res., 5, 126 (1964).

13) M. Kates, Adv. Lipid Res., 8, 225 (1970).

14) W. Fischer, E. Heinz and M. Zeus, Hoppe-Seyler's $Z$. Physiol. Chem., 354, 115 (1973).

15) D. I. Arnon, Plant Physiol., 24, 1 (1949).

16) H. P. Sieberz and E. Heinz, Z. Naturforsch., 32c, 193 (1977).

17) H. P. Sieberz, E. Heinz and L. Bergmann, Plant Sci. Lett., 12, 119 (1978).

18) J. Soimajarui and R. R. Linko, J. Agric. Food Chem., 27, 1279 (1979).

19) O. Hirayama and T. Suzuki, Agric. Biol. Chem., 32, 549 (1968).

20) M. Kates, A. C. Wilson and A. I. Roche, "Advances in the Biochemistry and Physiology of plant Lipids," ed. by L.-A. Appelqvisit and C. Liejenberg, Elsevier/North-Holland Biomedical Press, 1979 , p. 329.

21) B. M. Leese and R. M. Leech, Plant Physiol., 47, 789 (1976).

22) J. Bahl, B. Francke and R. Moneger, Planta, 129, 193 (1976).

23) J. Ohnishi and M. Yamada, Plant \& Cell Physiol., 21, 1595 (1980).

24) T. Matsuzaki, A. Koiwai and N. Kawashima, Agric. Biol. Chem., 45, 2379 (1981)

25) T. Matsuzaki, A. Koiwai and N. Kawahima, Plant \& Cell Physiol., 24, 193 (1983).

26) G. H. Schmid, Methods in Enzymol., 23, 175 (1971).

27) Y. Nakamura and M. Yamada, Plant \& Cell Physiol., 16, 163 (1975).

28) K. Nishida, F. Sato and Y. Yamada, Plant \& Cell Physiol., 21, 47 (1980).

29) J. S. Paul and J. A. Bassham, Plant Physiol., 60, 775 (1977).

30) W. Hüsemann, S. S. Radwan, H. K. Mangold and W. Barz, Planta, 147, 379 (1980). 